\title{
Source Redshifts from Gravitational-Wave Observations of Binary Neutron Star Mergers
}

\author{
C. Messenger, ${ }^{1, *}$ Kentaro Takami, ${ }^{2,3}$ Sarah Gossan, ${ }^{4}$ Luciano Rezzolla, ${ }^{3,2}$ and B. S. Sathyaprakash ${ }^{5}$ \\ ${ }^{1}$ SUPA, University of Glasgow, Glasgow G12 8QQ, United Kingdom \\ ${ }^{2}$ Max-Planck-Institut für Gravitationsphysik, Albert Einstein Institut, Am Mühlenberg 1, \\ 14476 Potsdam, Germany \\ ${ }^{3}$ Institut für Theoretische Physik, Max-von-Laue-Strasse 1, 60438 Frankfurt, Germany \\ ${ }^{4}$ TAPIR, California Institute of Technology, 1200 East California Boulevard, Pasadena, \\ California 91125, USA \\ ${ }^{5}$ School of Physics and Astronomy, Cardiff University, 5, The Parade, Cardiff CF24 3AA, United Kingdom
} (Received 22 January 2014; revised manuscript received 4 June 2014; published 8 October 2014)

\begin{abstract}
Inspiraling compact binaries as standard sirens will become an invaluable tool for cosmology when we enter the gravitational-wave detection era. However, a degeneracy in the information carried by gravitational waves between the total rest-frame mass $M$ and the redshift $z$ of the source implies that neither can be directly extracted from the signal; only the combination $M(1+z)$, the redshifted mass, can be directly extracted from the signal. Recent work has shown that for third-generation detectors, a tidal correction to the gravitational-wave phase in the late-inspiral signal of binary neutron star systems can be used to break the mass-redshift degeneracy. Here, we propose to use the signature encoded in the postmerger signal allowing the accurate extraction of the intrinsic rest-frame mass of the source, in turn permitting the determination of source redshift and luminosity distance. The entirety of this analysis method and any subsequent cosmological inference derived from it would be obtained solely from gravitational-wave observations and, hence, would be independent of the cosmological distance ladder. Using numerical simulations of binary neutron star mergers of different mass, we model gravitational-wave signals at different redshifts and use a Bayesian parameter estimation to determine the accuracy with which the redshift and mass can be extracted. We find that for a known illustrative neutron star equation of state and using the Einstein telescope, the median of the $1 \sigma$ confidence regions in redshift corresponds to $\sim 10 \%-20 \%$ uncertainties at redshifts of $z<0.04$.
\end{abstract}

DOI: 10.1103/PhysRevX.4.041004

\section{INTRODUCTION}

The prospects for gravitational-wave (GW) astronomy in the era of advanced detectors are promising, with several detections expected before the end of the decade when Advanced LIGO [1], Advanced Virgo [2], and KAGRA [3] become fully operational. Among the sources of GWs expected to be detected are the inspiral and coalescence of binary neutron stars (BNSs), neutron star-black hole binaries, and binary black holes. Population models suggest that the detection rate of compact binary coalescences for BNS will be $\sim 10 \mathrm{yr}^{-1}$, when Advanced LIGO [4] reaches its design sensitivity. The results presented in this paper concern GW detections made with third-generation detectors such as the Einstein telescope (ET) [5,6], which is expected to have detection rates $\sim 3$ orders of magnitude greater than advanced detectors for compact binary systems.

* Corresponding author.

christopher.messenger@glasgow.ac.uk

Published by the American Physical Society under the terms of the Creative Commons Attribution 3.0 License. Further distribution of this work must maintain attribution to the author(s) and the published article's title, journal citation, and DOI.
Subject Areas: Astrophysics, Cosmology, Gravitation

The inspirals of compact binary systems are also known as standard sirens [7], as their luminosity distance can be extracted from GW observations alone, without the need for any detailed modeling of the source or of the properties of the media (apart from strong or weak lensing) along the GW path. This is because the observed amplitude of GWs during the inspiral phase reaches the detector essentially unaltered and depends on a small number of parameters, which can all be measured using a network of GW detectors. These parameters include the total gravitational mass and mass ratio of the system, the spins of the compact objects, the orientation of the binary's orbital plane with respect to the line of sight, the source's position on the sky, and the luminosity distance to the source. GW observations can very accurately measure the signal's phase evolution, which depends only on the total mass and mass ratio of a binary. Simultaneously, a network of detectors can determine the sky position, GW polarization angle, orbital inclination, and the distance to the binary. The observed total mass, however, is not the system's intrinsic mass $M$ (i.e., mass as measured in the rest frame of the source) but the redshifted mass $M_{z} \equiv M(1+z)$. This is known as the mass-redshift degeneracy. 
The mass-redshift degeneracy is detrimental to the application of GW observations for cosmological inference. The relationship of the source's luminosity distance to its redshift on cosmological scales is precisely that which allows us to probe the parameters governing a cosmological model. Breaking the mass-redshift degeneracy requires an electromagnetic (EM) identification to tie the source to its host galaxy and thereby extract the source's redshift. It was thought, until recently, that there is no way to infer the source's redshift from GW observations alone.

The use of GW observations to extract information that is necessary for cosmography [e.g., estimation of the Hubble parameter and the dark energy equation of state (EOS)] and astrophysics [e.g., measurement of the masses and radii of neutron stars (NSs) and the EOS of matter at supranuclear densities] requires precision measurements of both the luminosity distance and the intrinsic mass of the source. The mass-redshift degeneracy forces reliance on EM identification of host galaxies [8-13], which may be possible only very rarely. For example, using gamma-ray bursts or the predicted EM afterglows of NS mergers [14] for identification of the host galaxy greatly reduces the available signal population for cosmography and could potentially lead to observational bias. For gamma-ray bursts this is because the emission is believed to be strongly beamed along a jet [15-17], while GW emission is expected to be approximately isotropic (quadrupolar), and hence, only a small fraction $\left(\sim 10^{-3}\right)$ of all GW events will have gamma-ray burst counterparts [10]. Determining the EM counterparts to binary-black-hole mergers is also a very active area of research and several simulations have already been performed in this context [18-22] to provide first estimates on the properties and energetics of these emissions.

Some authors have explored other approaches to measure cosmological parameters without the aid of EM counterparts. These methods fall into two categories. The first relies on the measurement of the redshifted mass distribution of BNS systems, which, combined with reasonable assumptions on the form and width of the intrinsic mass distribution, would yield an estimate of the Hubble constant [23-26]. A more robust approach [27], originally proposed by Schutz [7], uses error regions on the sky derived from multidetector GW observations to limit the possible number of host galaxies. The combination of multiple ( $\gtrsim 10) \mathrm{GW}$ events then resolves the apparent degeneracy between possible Hubble constant values.

In this paper, we draw attention to an additional feature of the BNS waveform caused by matter effects that can be exploited for cosmological inference by third-generation GW detectors. For BNS systems, there are two signatures in the GW signal from the late-inspiral and postmerger stages that depend on the rest-frame source mass and could potentially provide a measure of the source's intrinsic mass. Such a measurement would break the mass-redshift degeneracy and help return both the source redshift and the intrinsic total mass from GW observations alone. The first effect is concerned with the correction in the orbital phase due to tidal effects that appear at order $(v / c)^{10}$ beyond the leading order in the post-Newtonian approximation to Einstein's equations [28], where $v$ and $c$ are the orbital and light velocities, respectively. This is a secular effect that becomes important as the two bodies approach each other and was first proposed as a cosmological tool in Ref. [29]. The second effect occurs in the postmerger stage and causes a significant departure in the post-Newtonian evolution of the system. Unless the two stars are not very massive, the newly formed object is a hypermassive neutron star (HMNS) [30], which develops a bar-mode deformation, which can survive even for a fraction of a second (cf. Fig. A1 of Ref. [31]), delaying the birth of the black hole and emitting GWs in a narrow frequency range. The importance of this effect has not yet been considered for cosmological exploitation.

In this paper, we propose to use the GW signal including the inspiral phase and the HMNS signature to extract the intrinsic gravitational masses and the source redshift. The power spectrum of the HMNS stage of the merger of BNS systems has been shown to contain prominent spectral features that vary smoothly in frequency with the total gravitational mass of the system $[32,33]$. The inspiral stage of the waveform can be used to obtain a highly accurate measurement of the redshifted total mass of the system $M_{z}$. This allows us to constrain the true values of the rest mass and redshift to a relatively narrow band spanning the full range of the $(z, M)$ plane (cf. Fig. 4). Independently, the HMNS stage of the waveform allows us to measure the redshifted fundamental frequencies of two prominent spectral features to a reasonable accuracy. Using these and an empirically determined relationship between the total gravitational mass of the binary and the rest-frame fundamental frequencies, we are then able to independently constrain a second region of the $(z, M)$ plane (cf. Fig. 4). The localized intersection of the two regions in the $(z, M)$ plane allows us to break the mass-redshift degeneracy present in both measurements and make an estimate of the redshift and gravitational mass of the system. An illustration of this idea is shown in Fig. 1, which can be directly compared to an example of the results of this analysis shown in Fig. 4.

The detectability of the HMNS part of the waveform is not likely to be high for sources observed in advanced detectors, as these features lie at frequencies significantly higher than the sensitive bands of ground-based detectors. We, therefore, explore how we might use the signature of HMNS in the context of the ET, a third-generation groundbased interferometric GW detector $[5,6]$. We also highlight that the analysis we present here depends on the valid assumption that the NS EOS will be known accurately via various methods, including direct GW detection by advanced GW detectors by the third-generation GW 


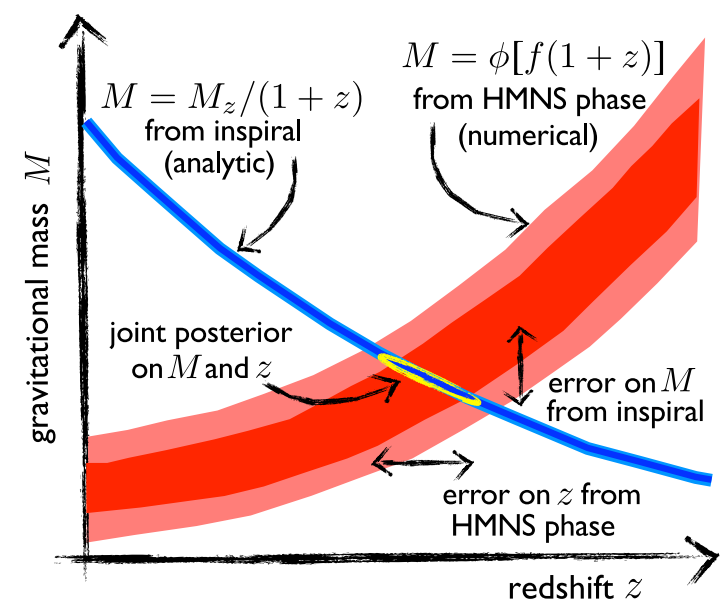

FIG. 1. Illustration of how the mass-redshift degeneracy is broken through the use of information from the inspiral and HMNS stage of a BNS merger event. Information on the redshifted mass as a function of the redshift (blue stripe) can be correlated with complementary information from the spectral properties of the HMNS phase. The overlap will provide a localized range in mass and redshift, breaking the degeneracy.

detector era. It was shown in Refs. [32,34] that the determination of the peak frequency in the postmerger GW signal from a single source will constrain the NS EOS significantly. It has not been widely discussed in the literature that in order to do this accurately a host galaxy must be associated with the source and its redshift measured so as to infer the unredshifted mass. It is likely that at least a small number of BNS detections from advanced detectors will be sufficient to constrain the EOS, which will then be improved upon by thirdgeneration detectors. It is expected that a population of BNS events observed by advanced GW detectors will be able to measure the EOS quite accurately [35].

The statistical measurement uncertainties in redshift quoted in this work neglect systematic uncertainties that could arise due to poorly constrained EOS, in particular, the dependence of the peak frequencies on the total mass of the merged object. Such uncertainties could lead to a bias in redshift measurement that might be comparable to the statistical uncertainties if it is not possible to accurately constrain the EOS on the time scale of the ET. We expect, however, that these uncertainties will be sufficiently resolved by the time of the ET: It should be possible to establish an accurate relationship between the mass and postmerger spectral frequencies both by observations of nearby events by advanced detectors and the ET and by more advanced numerical simulations. Finally, we note that measurements by $\mathrm{x}$-ray satellites, such as the large observatory for $\mathrm{x}$-ray timing (LOFT), could also provide stringent constraints on the NS EOS by the measurement, using three complementary types of pulsations, of mass and radius of at least four NSs with an instrumental accuracy of $4 \%$ in mass and $3 \%$ in radius [36].

The rest of the paper is organized as follows: In Sec. II, we describe the numerical waveforms used for this analysis. In Sec. III, we describe our robust, but ad hoc, parametrization and modeling of the HMNS power spectrum. In Sec. IV, we describe the analysis methods used to simulate and measure the HMNS spectral features. We then describe the procedure with which these measurements are combined to obtain the redshift and gravitational masses of the source. Finally, in Sec. V, we conclude with discussions of our results and future directions for this research.

\section{NUMERICAL SIMULATIONS OF BNS SYSTEMS}

All of our calculations were performed in full general relativity. The evolution of the spacetime is obtained by using the CCATIE code, a finite-differencing code providing the solution of a conformal traceless formulation of the Einstein equations [37], with a " $1+\log$ " slicing condition and a "Gamma-driver" shift condition. The generalrelativistic hydrodynamics equations are solved using the WHISKY code $[30,38]$, with the Marquina flux formula and a piecewise parabolic method (PPM) reconstruction. For the sake of simplicity, we model the NS matter as an ideal fluid with a gamma-law EOS, $p=(\Gamma-1) \rho \epsilon$ with $\Gamma=2$, where $p$ is the pressure, $\rho$ the rest-mass density, and $\epsilon$ specific internal energy (see Ref. [39] for details). The grid hierarchy, with a reflection symmetry condition across the $z=0$ plane and a $\pi$-symmetry condition across the $x=0$ plane, is handled by the CARPET mesh refinement driver [40], where we use six refinement levels and the spacing of

TABLE I. Properties of our initial data of equal-mass BNSs with the initial coordinate separation $45 \mathrm{~km}$. Reported in the various columns are the baryon mass $M_{b}$ of each star, the Arnowitt-Deser-Misner (ADM) mass $M_{\mathrm{ADM}}$ of the system at initial data, the gravitational mass $M_{\infty}$ of each star at infinite separation $\left(M=2 M_{\infty}\right)$, the circumferential radius $R_{\infty}$ of each star at infinite separation, the compactness $\mathcal{C} \equiv M_{\infty} / R_{\infty}$, and the orbital frequency $f_{\text {orb }}$ at the initial separation.

\begin{tabular}{lccccc}
\hline \hline$M_{b}\left[M_{\odot}\right]$ & $M_{\mathrm{ADM}}\left[M_{\odot}\right]$ & $M_{\infty}\left[M_{\odot}\right]$ & $R_{\infty}\left[G M_{\odot} / c^{2}\right]$ & $\mathcal{C}$ & $f_{\text {orb }}[\mathrm{Hz}]$ \\
\hline 1.4237 & 2.6578 & 1.3413 & 11.386 & 0.11781 & 281.80 \\
1.4662 & 2.7305 & 1.3784 & 11.276 & 0.12224 & 284.62 \\
1.5099 & 2.8049 & 1.4163 & 11.158 & 0.12693 & 287.45 \\
1.5549 & 2.8811 & 1.4550 & 11.031 & 0.13190 & 290.29 \\
1.5947 & 2.9478 & 1.4890 & 10.914 & 0.13643 & 292.74 \\
\hline \hline
\end{tabular}


the finest grid is $0.15 G M_{\odot} c^{-2} \sim 0.221 \mathrm{~km}$. We extract the GWs, consisting of a plus and cross polarization and sampled in time at a rate of $\Delta t=1.68 G M_{\odot} c^{-3} \sim 8.27 \times 10^{-3} \mathrm{~ms}$, equivalent to a sampling rate of $\sim 121 \mathrm{kHz}$, at a distance $R_{0}=500 G M_{\odot} c^{-2} \sim 738 \mathrm{~km}$. We analyze only the $\ell=$ $m=2$ mode of GWs, which is the dominant one. As the initial data, we use quasiequilibrium irrotational BNSs generated by the multidomain spectral-method code LORENE [41] under the assumption of a conformally flat spacetime metric. We consider five equal-mass binaries with an initial coordinate separation of the stellar centers of $45 \mathrm{~km}$ and polytropic EOS, $p=K \rho^{\Gamma}$ with an adiabatic exponent $\Gamma=2$ and polytropic constant $K=123.6$ (in units $c=$ $G=M_{\odot}=1$ ); details of the different binaries are shown in Table I. A very important requirement of our sample of BNSs is that they are only very finely separated in total gravitational mass, with differences that are of the order of $2 \%$ only. Producing such a sample at a fixed separation is far from trivial and has represented a major numerical difficulty, stretching the capabilities of the LORENE libraries. Once evolved, the stars perform approximately 3.5 orbits before merger.

\section{FREQUENCY DOMAIN MODELING OF THE HMNS}

In order to perform the parameter estimation described in Sec. IV, we must first be able to parametrize and model the HMNS stage of the waveform. Using our five waveforms as a basis, the current state-of-the-art numerical simulations of BNS systems do not yet give us the insight and accuracy required to model the phase evolution of the HMNS waveform as a function of the system's mass. This, coupled with the assumption that there exists a smooth relationship between the total gravitational mass of the system $M$ and the frequencies of prominent spectral features, forces us to model the signal power rather than the complex waveform. Therefore, in our HMNS analysis, we are insensitive to information encoded in the phasing of the waveform. Unless a semianalytic description of the phase evolution in the HMNS stage is possible; the one adopted here is probably the only approach feasible.

For each numerical waveform, we perform the following procedure in order to compute noise-free power-spectrum reference templates.

The time series for both the plus and the cross polarizations are preprocessed using a fifth-order highpass Butterworth filter with knee frequency at $1 \mathrm{kHz}$. A symmetric time-domain Tukey window with $\alpha$ parameter 0.25 (affecting the first and last $4 \mathrm{~ms}$ of the time series) is then applied. This is done to suppress the leakage of power from the last few cycles of the inspiral and initial merger stage of the waveform and to cut off the waveform before the system collapses to a black hole. The discrete Fourier transform is then computed for each polarization from which we construct the reference template

$$
\mathcal{T}(f) \equiv \frac{\left|\tilde{h}_{+}(f)\right|^{2}+\left|\tilde{h}_{\times}(f)\right|^{2}}{S_{h}(f)} \cong \frac{2\left|\tilde{h}_{+}(f)\right|^{2}}{S_{h}(f)}
$$

where $S_{h}(f)$ is the noise spectral density of the detector, which we choose to be that of the ET-B [42] design [43]. Note that since we are taking the signal power, and the preprocessing steps suppress frequency-domain artifacts from the finite duration signals, the plus and cross power contributions are approximately equal. We make no assumptions regarding the polarization or source-detector orientation of a potential signal in this construction. For the ET colocated detectors, arbitrary values of these parameters serve only to scale the overall amplitude of the waveform.

Visual inspection of these reference templates as a function of frequency, shown in Fig. 2, allows us to clearly identify the two primary spectral features of interest. The first feature, at frequencies $\approx 1.2-1.6 \mathrm{kHz}$, is approximately Gaussian in profile and moves to higher frequencies for higher mass systems. In contrast, the second feature, at frequencies $\approx 1.7-3 \mathrm{kHz}$, appears to be best described by a sloping trapezoid with rounded shoulders and a central frequency and bandwidth that also grows with increasing system mass. In addition, there appears to be a third power component at low frequencies, i.e., $\leq 2 \mathrm{kHz}$, that becomes more dominant as the system mass increases. A reasonable approximation to this third feature is a second Gaussian of lower amplitude and greater variance than that used to model the first feature. For the purposes of this work, this third feature is included only to improve the quality of our model fitting. Mathematically, our entire ad hoc model of the waveform power spectrum can be expressed as

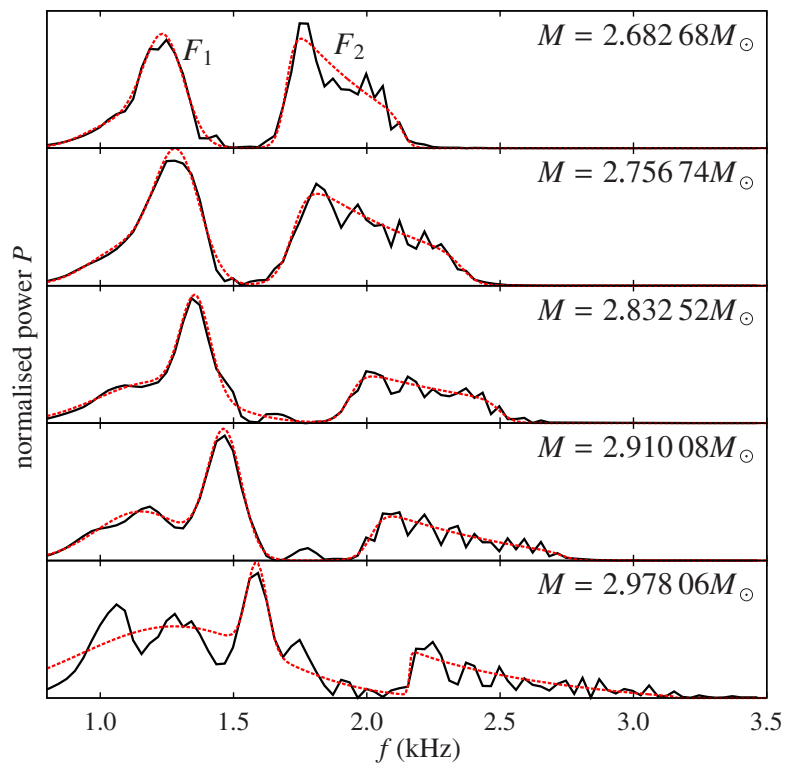

FIG. 2. The normalized power-spectrum reference templates [Eq. (1)] for each of the five system masses as a function of frequency (black lines). Also plotted are the best-fit model templates defined in Eq. (2) (red dashed lines). 


$$
\begin{aligned}
\mathcal{S}(f ; \lambda)= & S_{h}^{-1}(f)\left[A_{1} e^{-\left(f-F_{1}\right)^{2} / W_{1}^{2}}+A_{3} e^{-\left(f-F_{3}\right)^{2} / W_{3}^{2}}\right. \\
& \left.+A\left(f ; A_{2 a}, A_{2 b}, F_{2}, W_{2}\right) \gamma\left(f ; F_{2}, W_{2}, s\right)\right],
\end{aligned}
$$

where $A_{1}$ and $A_{3}$ are amplitude terms with $F_{1}, F_{3}$ and $W_{1}, W_{3}$ as central frequencies and half-widths (standard deviations), respectively, of the Gaussian features. The template is whitened using the detector noise spectral density as is done for the reference template. We also define

$$
\begin{aligned}
A\left(f ; A_{2 a}, A_{2 b}, F_{2}, W_{2}\right) \equiv & \frac{1}{2 W_{2}}\left[\left(A_{2 b}-A_{2 a}\right)\left(f-F_{2}\right)\right. \\
& \left.+W_{2}\left(A_{2 b}+A_{2 a}\right)\right],
\end{aligned}
$$

which is a linear slope of amplitude $A_{2 a}$ for $f=F_{2}-W_{2}$ and amplitude $A_{2 b}$ for $f=F_{2}+W_{2}$. Finally, the function

$$
\gamma\left(x ; F_{2}, W_{2}, s\right) \equiv \frac{1}{1+e^{-\left(f-F_{2}+W_{2}\right) / s}}-\frac{1}{1+e^{-\left(f-F_{2}-W_{2}\right) / s}}
$$

is the difference between two simple sigmoid functions, which serves to bound our model of the second spectral feature component between the frequencies $F_{2} \pm W_{2}$ with a smooth transition from zero to unity over a fixed frequency range controlled by the parameter $s$. This $a d$ hoc template is therefore described by the 11-dimensional parameter vector $\lambda \equiv\left(A_{1}, A_{2 a}, A_{2 b}, A_{3}, F_{1}, F_{2}, F_{3}, W_{1}\right.$, $\left.W_{2}, W_{3}, s\right)$.

We employ a simple least-squares fitting procedure to obtain our best-fit parameters $\lambda^{\prime}(M)$ for each system mass. In all cases, there are restrictions on the allowed parameter space ensuring that (a) $A_{2 a}>A_{2 b}$, such that the slope of the second spectral feature is negative, (b) $F_{3}<F_{2}$, such that the third (Gaussian) spectral feature is restricted to the lower frequencies, and (c) the smoothing length $s<W_{2} / 5$, such that the smoothed transition regions of the second feature account for less that $10 \%$ of the total feature width. As can be seen from Fig. 2, our choice of model and parameter restrictions provides a reasonable fit to the numerical data across our range of masses. We note that the quality of fit does begin to deteriorate at higher masses. This is due to the fact that, as the mass of the binary increases, the HMNS is further away from a stable equilibrium and its dynamics is much more violent; in particular, the bar-deformed object rapidly spins up via the copious emission of GWs, leading to a very broad spectrum for the $F_{2}$ frequency.

The model fit depends on the parameter set $\lambda$, but we are only truly interested in a measure of the characteristic frequencies corresponding to the two dominant spectral features. For the lower-frequency Gaussian feature, we choose to use the central frequency of the corresponding fit $F_{1}$ as this measure. For the higher-frequency, less symmetric, second feature, we choose to define its characteristic frequency as the average frequency within the bandwidth of the second feature weighted by our best-fit power model. This choice is made in an attempt to more robustly track the location of the power of the second feature. Hence, the lower and upper characteristic frequencies are defined as

$f_{1} \equiv F_{1}$,

$f_{2} \equiv \frac{\int_{0}^{\infty} A\left(f ; A_{2 a}^{\prime}, A_{2 b}^{\prime}, F_{2}^{\prime}, W_{2}^{\prime}\right) \gamma\left(f ; F_{2}^{\prime}, W_{2}^{\prime}, s^{\prime}\right) f d f}{\int_{0}^{\infty} A\left(f ; A_{2 a}^{\prime}, A_{2 b}^{\prime}, F_{2}^{\prime}, W_{2}^{\prime}\right) \gamma\left(f ; F_{2}^{\prime}, W_{2}^{\prime}, s^{\prime}\right) d f}$

Taking the best-fit parameters for each system mass and computing the corresponding $f_{1}$ and $f_{2}$ values, we indeed validate the initial hypothesis that there is a smooth relationship between these values and the total gravitational mass of the system. This relationship is shown in Fig. 3, where we plot total gravitational mass versus $f_{1}$ and $f_{2}$. Also plotted are the following best-fit second and firstorder polynomials for $f_{1}$ and $f_{2}$, respectively, and whose expressions in $\mathrm{Hz}$ are

$$
\begin{aligned}
& f_{1}(M)=1331+992 \Delta M+2538 \Delta M^{2}, \\
& f_{2}(M)=2087+2018 \Delta M,
\end{aligned}
$$

where $\Delta M / M_{\odot} \equiv M / M_{\odot}-2.8$. In all cases, the residuals from this polynomial fit are $<50 \mathrm{~Hz}$. These functions represent our empirically determined relationship between

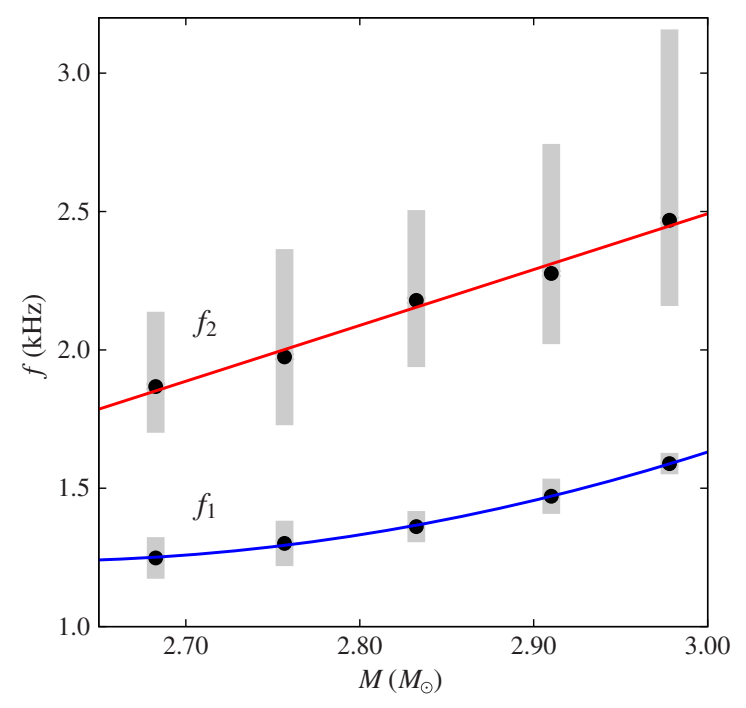

FIG. 3. Measured best-fit values of $f_{1}$ and $f_{2}$ versus the total system mass (solid black circles). The vertical gray bars on the lower-frequency spectral feature represent the standard deviation of the Gaussian fit at each mass value (not the measurement uncertainty). The corresponding bars on the higher-frequency data represent the total frequency span of the feature. The blue curves and the red curves show the least-squares fit to $f_{1}$ and $f_{2}$ for a second- and first-order polynomial, respectively [given in Eq. (6)]. 
the characteristic frequencies of the two spectral features and the total gravitational mass. The phenomenological frequencies so obtained essentially track the eigenfrequencies of the HMNS, which has recently been shown to behave as an isolated, self-gravitating system, whose dynamics can be described as a superposition of different oscillation modes (see Ref. [44] for details).

Some remarks are worth making. First, Eq. (6) is clearly tuned to our choice of EOS, but equivalent expressions can be derived for any EOS [33]. Second, the functional dependence of the frequencies on the total mass of the system is only weakly dependent on the mass ratio; as a result, although our simulations refer to equal-mass systems, we expect the functions (6) to also be a good approximation for unequal-mass binaries (see also Ref. [32], where this is discussed in detail). Finally, as anticipated in the Introduction, the importance of the relations (6) is that they can be employed to break the mass-redshift degeneracy.

\section{ANALYSIS OF THE DATA}

We now describe how we simulate the occurrence and subsequent measurement of BNS signals in thirdgeneration GW detectors. We separate this process into two parts. The first is the simulation and measurement of the redshifted characteristic frequencies of the HMNS spectral features in the presence of Gaussian detector noise. The second is the independent measurement of redshifted mass parameters using the inspiral stage of a BNS waveform. We then show how these measurements can be combined to infer the gravitational mass and redshift of a source using the results of the previous section.

\section{A. Measurement of the HMNS phase}

The numerical waveforms comprise time series of the plus and cross polarizations of the GW signal at a distance of $R_{0}=500 M_{\odot}$, which we label $h_{+}^{(0)}(t)$ and $h_{\times}^{(0)}(t)$, respectively. Using these data, we are able to simulate waveforms from BNS systems with arbitrary orientations, sky position, GW polarization, phase, and redshift.

We first compute the relarizations

$$
\begin{aligned}
& \tilde{h}_{+}(f, z) \equiv \frac{R_{0}}{D_{L}(z)} \tilde{h}_{+}^{(0)}[f(1+z)], \\
& \tilde{h}_{\times}(f, z) \equiv \frac{R_{0}}{D_{L}(z)} \tilde{h}_{\times}^{(0)}[f(1+z)],
\end{aligned}
$$

where $D_{L}$ is the luminosity distance and is a function of the redshift $z$ and a choice of cosmological parameters. We use a standard cosmological model described by the parameters $h_{0}=0.71, \Omega_{m}=0.27$, and $\Omega_{\Lambda}=0.73$. In order to evaluate these waveforms at arbitrary redshifted frequencies we use a cubic spline interpolation scheme on the overresolved discrete Fourier transform of the original time series data.

To simulate GWs with arbitrary sky positions, inclination, and polarization parameters, we take the numerically generated plus and cross waveforms and apply antenna response and inclination-dependent functions such that the signal at the detector is

$$
\begin{aligned}
\tilde{h}_{i}(f)= & {\left[\frac{1}{2} F_{i}^{(+)}(\phi, \theta, \psi)\left(1+\mu^{2}\right) \tilde{h}_{+}(f)\right.} \\
& \left.+F_{i}^{(\times)}(\phi, \theta, \psi) \mu \tilde{h}_{\times}(f)\right] e^{-i \varphi},
\end{aligned}
$$

where $\phi, \theta, \psi$ are the source right ascension, declination, and polarization angles, respectively. The cosine of the inclination angle is given by $\mu$ and we apply an arbitrary constant phase factor $\varphi$. Explicit definitions of the antenna patterns $F_{i}^{(+/ \times)}$, for the ET detector (where $i$ indexes the three ET interferometers) can be found in Ref. [12]. Independent Gaussian noise $\tilde{n}_{i}(f)$ of spectral amplitude matching the ET-B noise curve is then added to the frequency-domain waveforms corresponding to each ET interferometer. The simulated data are then

$$
\tilde{d}_{i}\left(f_{k}\right)=\tilde{h}_{i}\left(f_{k}\right)+\tilde{n}_{i}\left(f_{k}\right),
$$

where $i$ indexes the ET interferometers and $k$ the discrete frequency grid on which the waveform has been evaluated. This grid is defined by the length and sampling time of the original numerical waveforms such that the frequency resolution $\Delta f=(N \Delta t)^{-1}=31.2118 \mathrm{~Hz}$ and only the 86 frequencies between 800 and $3.5 \mathrm{kHz}$ are included.

At this point, we apply a fifth-order high-pass Butterworth filter to the data with knee frequency $1 \mathrm{kHz}$ and a timedomain Tukey window with $\alpha$ parameter 0.25 . We therefore treat the data in exactly the same way as is done in the template generation procedure and for the same reasons, namely, to minimize contributions to the signal power from the last few cycles of the inspiral and merger. It is also important to perform this filtering after the waveform has been redshifted and noise has been added, since in this way, any spectral features resulting from this preprocessing will not show any artificial cosmological dependence.

We define the detector noise weighted power as

$$
P\left(f_{k}\right) \equiv 4 \sum_{i=1}^{3} \frac{\left|\tilde{d}_{i}\left(f_{k}\right)\right|^{2}}{S_{h}^{(i)}\left(f_{k}\right)} \Delta f .
$$

At any given frequency, this power is governed by a noncentral $\chi^{2}$ distribution with 6 degrees of freedom. The noncentrality parameter of this distribution is given by the squared optimal SNR in that frequency bin,

$$
\rho^{2}\left(f_{k}\right) \equiv 4 \sum_{i=1}^{3} \frac{\left|\tilde{h}_{i}\left(f_{k}\right)\right|^{2}}{S_{h}^{(i)}\left(f_{k}\right)} \Delta f \cong \mathcal{S}\left(f_{k} ; \lambda\right),
$$


which is approximately equal to our power-spectrum template defined by Eq. (2). We note that the freedom in our choice of amplitude parameters in the template allows us to use an equality rather than a proportionality in the above relationship. It then follows that the likelihood can be written as

$$
\begin{aligned}
p(\{P\} \mid \lambda)= & \prod_{k=1}^{L}\left\{\frac{P\left(f_{k}\right)}{2 \mathcal{S}\left(f_{k}, \lambda\right)} e^{-\left[\mathcal{S}\left(f_{k}, \lambda\right)+P\left(f_{k}\right)\right] / 2}\right. \\
& \left.\times I_{2}\left[\sqrt{P\left(f_{k}\right) \mathcal{S}\left(f_{k}, \lambda\right)}\right]\right\}
\end{aligned}
$$

where $I_{2}$ is the second-order modified Bessel function of the first kind and $L$ is the total number of frequency bins. We note that our template is now sensitive to the redshifted frequencies present in the data and not the intrinsic frequencies. Therefore, we define a redshifted parameter vector $\lambda_{z}$ containing the same amplitude parameters as $\lambda$, but with the frequencies $F_{j, z} \equiv F_{j} /(1+z), W_{j, z} \equiv W_{j} /$ $(1+z)$, and $s_{z} \equiv s /(1+z)$. Consequently, our parameters of interest become the redshifted characteristic frequencies $f_{j, z} \equiv f_{j} /(1+z)$.

Next, we apply a nested-sampling algorithm [45] to the data to provide samples from the posterior distributions of the $\lambda_{z}$ parameter set. We assume uniform priors on all parameters and use output posterior samples to generate posterior samples of the redshifted frequencies $f_{1, z}$ and $f_{2, z}$. These samples represent the posterior probability distribution function (PDF) $p\left(f_{j, z} \mid\{P\}\right)$. We keep the prior parameter ranges of $\lambda_{z}$ constant for all simulations and, therefore, treat each simulation identically, independent of system mass and redshift. The range of our priors are given in Table II, where we also define the additional constraints that the amplitude of the first feature must be greater than that of the third feature, the slope of the second feature must be negative, and, finally, the smoothing length $s_{z}$ must be less than $10 \%$ of the total width of the second feature.

The resultant typical measurement uncertainties in $f_{1}$ and $f_{2}$ for each simulated system as a function of redshift are given in Table III. These intermediate results show, as expected, that the accuracy of measurement (reported in brackets) is $\mathcal{O}$ (few\%) and decreases with increasing distance. It is also clear that there is a mild trend toward higher

TABLE II. The range of priors of the model parameters used in the calculation of the posterior distributions on $\lambda_{z}$. Additional prior constraints defined by $A_{1, z}>A_{3, z}, A_{2 a}>A_{2 b}$, and $s_{z}<$ $W_{2, z} / 5$ are also applied.

\begin{tabular}{lrrrrcccccr}
\hline \hline & $A_{1}$ & $A_{2 a}$ & $A_{2 b}$ & $A_{3}$ & $F_{1, z}$ & $F_{2, z}$ & $F_{3, z}$ & $W_{1, z}$ & $W_{2, z}$ & $W_{3, z}$ \\
\hline Min & 0 & 0 & 0 & 0 & 1100 & 1700 & 800 & 30 & 150 & 30 \\
Max & 100 & 100 & 100 & 100 & 1600 & 2700 & 1500 & 150 & 500 & 500 \\
\hline \hline
\end{tabular}

percentage uncertainties in higher-mass systems and that the percentage errors in frequency are comparable between the two spectral features.

\section{B. Measurement of the inspiral phase}

We treat the measurement of the inspiral stage of the waveform separately from the numerical simulations of the HMNS stage. At the redshifts relevant for the ET detector, the SNR from a BNS inspiral signal is

$$
\langle\rho\rangle \approx 80 z^{-1}, \quad \text { for } z<1,
$$

after averaging over sky position, polarization, and inclination angles. We ignore the use of tidal information in the inspiral stage for redshift inference (we discuss this in Sec. V). Measurement of just the inspiral phase of the signal allows us to infer the redshifted total mass $M_{z}$, but not the gravitational mass or redshift separately. Given that the SNR is going to be very high, the redshifted-mass parameters will be very well constrained from the inspiral phase alone. To quantify the accuracy of this measurement, we use a Fisher-matrix approach, which is a good approximation when the SNR is large and identical to that used in previous work on GW parameter estimation $[46,47]$. We consider as our signal model the frequencydomain stationary phase approximation of a nonspinning BNS inspiral signal, correct to 3.5 post-Newtonian order. It follows that the redshifted chirp mass $\mathcal{M}_{z} \equiv M_{z} \nu^{3 / 5}$ and symmetric mass ratio $\nu \equiv m_{1} m_{2} / M^{2}$, where $m_{1}$ and $m_{2}$ are the component masses, have fractional measurement uncertainties of $\Delta \mathcal{M}_{z} / \mathcal{M}_{z} \approx 5 \times 10^{-6}(z / 0.1)$ and $\Delta \nu / \nu \approx$ $10^{-3}(z / 0.1)$ for $z<1$. This corresponds to a fractional uncertainty in the redshifted total mass of

$$
\frac{\Delta M_{z}}{M_{z}} \approx \alpha 10^{-3}\left(\frac{z}{0.1}\right)
$$

which is dominated by the uncertainty in the symmetric mass ratio of the system. In subsequent use of this expression, we do not assume that the system consists of equal component masses. We also include a constant scale factor $\alpha$ and take a conservative approach by setting it equal to 10 , therefore overestimating the measurement uncertainty of the redshifted total mass.

In contrast to the HMNS stage, our analysis of the inspiral stage is quite simplistic. Given one of our original set of five numerically evolved binary systems and a choice of redshift, we assume a Gaussian uncertainty in the measurement of $M_{z}$ based on our Fisher-matrix result. We then draw a random Gaussian variable $M_{z}^{\prime}$, with mean equal to the original redshifted total mass and a standard deviation governed by Eq. (14) with $\alpha=10$, where a prime stands for the measured mass, which includes the measurement uncertainty, as opposed to the true redshifted total mass $M_{z}$. The corresponding likelihood function is then a 
TABLE III. The absolute measurement uncertainties in the redshifted characteristic frequencies of the dominant HMNS spectral features. The corresponding percentage uncertainties are given in brackets. We show pairs of results in units of $\mathrm{Hz}$ for $f_{1}$ (upper rows) and $f_{2}$ (lower rows) for each redshift and for each of the five total gravitational masses. Each value represents half of the span of the $68 \%$ confidence region on the frequency measurement averaged over 100 different noise realizations, source, and sky orientations.

\begin{tabular}{lcccrr}
\hline \hline \multirow{2}{*}{$z$} & \multicolumn{5}{c}{ Total gravitational mass $M\left(M_{\odot}\right)$} \\
\cline { 2 - 6 } 0.01 & 2.6827 & 2.7567 & 2.8325 & \multicolumn{1}{c}{2.9101} & 2.9781 \\
\hline \multirow{2}{*}{$0.02(0.6)$} & $7.74(0.6)$ & $5.82(0.4)$ & $5.03(0.3)$ & $6.03(0.4)$ \\
& $4.80(0.3)$ & $8.10(0.4)$ & $10.22(0.4)$ & $17.61(0.7)$ \\
0.03 & $11.84(1.0)$ & $14.59(1.1)$ & $13.87(1.0)$ & $11.74(0.8)$ & $16.26(1.0)$ \\
& $12.87(0.7)$ & $18.66(1.0)$ & $25.37(1.2)$ & $30.87(1.4)$ & $52.54(2.2)$ \\
0.04 & $19.91(1.6)$ & $22.50(1.8)$ & $22.91(1.7)$ & $22.72(1.6)$ & $47.93(3.1)$ \\
& $26.48(1.5)$ & $33.60(1.7)$ & $51.82(2.5)$ & $55.87(2.5)$ & $101.56(4.3)$ \\
& $35.09(2.9)$ & $38.77(3.1)$ & $34.47(2.6)$ & $37.21(2.6)$ & $67.41(4.4)$ \\
\hline \hline
\end{tabular}

Gaussian with the same standard deviation and mean equal to the randomly drawn $M_{z}$ value.

\section{Inference in the $(z, M)$ plane}

Starting with the measurement of the inspiral phase described in the previous section, we write the joint posterior PDF of the total gravitational mass and redshift as

$$
p\left(z, M \mid M_{z}^{\prime}\right) \propto \exp \left[-\frac{\left(M_{z}^{\prime}-M(1+z)\right)}{2\left(\Delta M_{z}\right)^{2}}\right]
$$

In the $(z, M)$ plane, this defines a narrow "stripe" of probability spanning a region that extends from high masses at low redshift to low masses at high redshift (see the negatively sloped blue curve in Fig. 1). We assume flat prior distributions for $M$ and $z$.

For the HMNS measurement, the following procedure is applied to each spectral feature indexed using $j$. We note that any given redshift and total gravitational mass of a BNS system defines a specific point in the $(z, M)$ plane. From Eq. (6), we can determine the intrinsic characteristic frequency of the spectral feature in question at this value of $M$. We can also calculate the corresponding redshifted characteristic frequency using the relation $f_{j, z}=f_{j} /$ $(1+z)$. The probability density associated with obtaining any particular value of $f_{j, z}$, hence, that particular $(z, M)$ pair, is given by the marginalized posterior PDF, $p\left(f_{1, z} \mid\{P\}\right)$, obtained from our analysis of the HMNS data described in Sec. IVA. We can, therefore, write

$$
p_{j}(z, M \mid\{P\}) \propto p\left(f_{j, z}=\frac{f_{j}(M)}{(1+z)} \mid\{P\}\right) .
$$

This function describes an arc of probability in the $(z, M)$ plane that sweeps almost orthogonally to that obtained from the measurement of the inspiral phase. An increase in the observed redshifted frequency can be obtained via either an increase in the total mass of the binary or a decrease in redshift of the source. Consequently, a highredshift high-mass system will generally have similar redshifted characteristic frequencies to a low-redshift low-mass system.

Combining the information from both spectral features and the inspiral phase of the signal and assuming statistical independence, the final joint posterior distribution of $z$ and $M$ is simply the product of all three distributions:

$p\left(z, M \mid M_{z}^{\prime},\{P\}\right) \propto p\left(z, M \mid M_{z}^{\prime}\right) \prod_{j=1}^{2} p_{j}(z, M \mid\{P\})$.

We show examples of joint posterior probabilities of $z$ and $M$ for all five systems studied, and for multiple redshift values, in Fig. 4. These examples mirror the original conceptual sketch in Fig. 1, where we can see how, in practice, the mass-redshift degeneracy is broken through the use of the spectral properties of the HMNS GW signal. For sources at redshifts $z=0.01-0.04$, the uncertainty in the measurement of the redshift is $\Delta z \sim$ $10 \%-20 \%$, over the full range of simulated system masses. In addition, the gravitational mass can be measured with fractional errors of $<1 \%$ in all cases. It can be seen that the choice of overestimating the total mass uncertainty by using a scale factor $\alpha=10$ does not strongly influence our results. The joint posteriors shown in Fig. 4 indicate that, due to the correlation between redshift and chirp mass, had the inspiral measurement been more accurate, the marginalized results on total gravitational mass and redshift would be only slightly improved.

In Tables IV and V, we give representative values for the fractional uncertainty on the measured redshift and total 

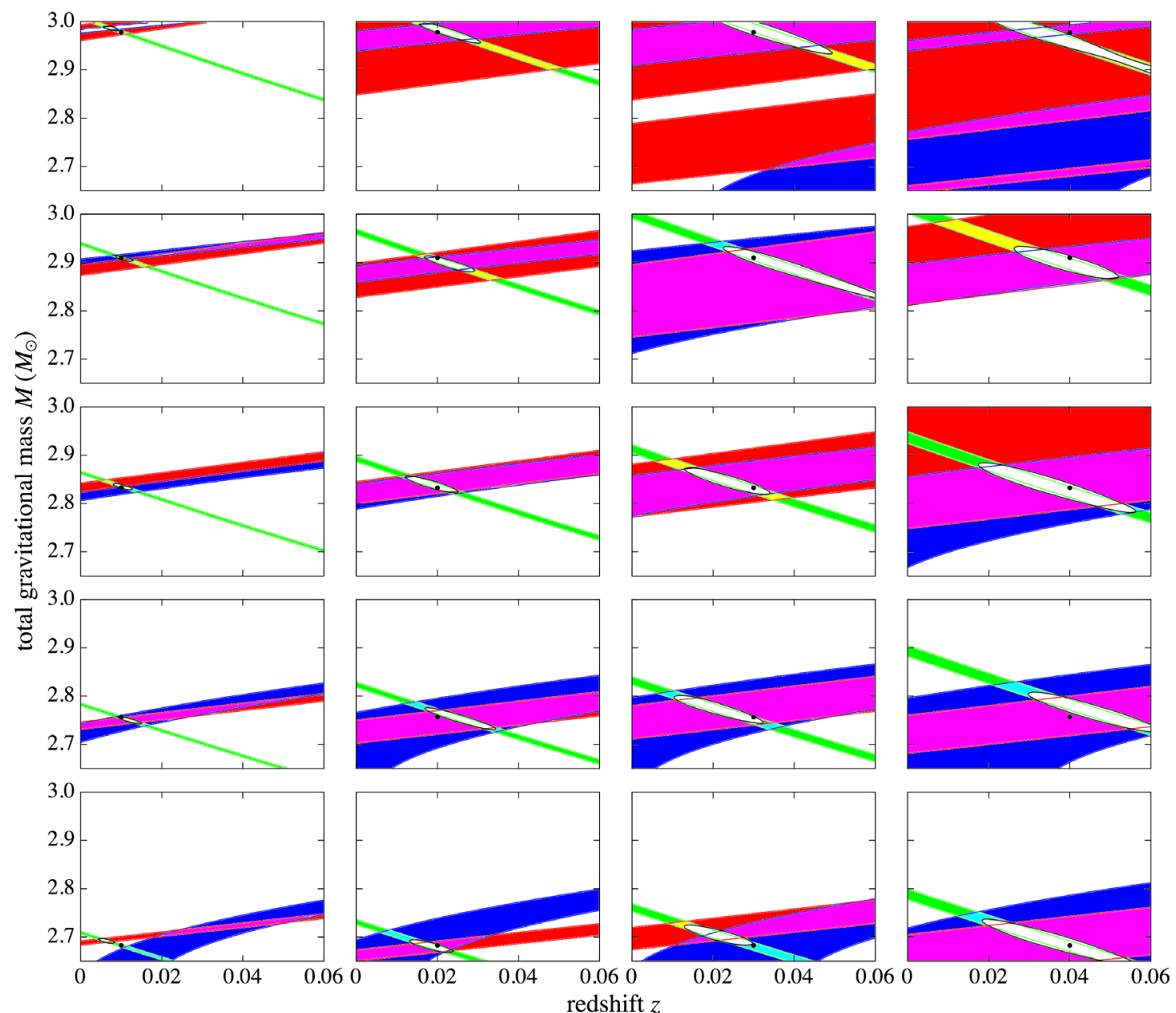

FIG. 4. The joint posterior distributions on the redshift and total gravitational mass of the BNS system for single representative realizations of noise and system parameters. Each row of plots represents a simulated signal of one of the five system masses (see Table I) ranging from low (bottom row) to high (top row) mass. Columns represent different simulated redshifts ranging from 0.01 (left) to 0.04 (right) in steps of 0.01. The green, blue, and red contours represent the posterior contributions from the inspiral measurement, the first HMNS spectral feature, and the second HMNS spectral feature, respectively. The black contours represent the final posterior distribution combining all measurements, and the black dots indicate the true simulated redshift and total mass values. In all cases, the contours enclose $68 \%$ of the probability. Overlapping regions have been filled according to the additive color system with the exception that regions outside all contours and the full interior of the final posterior contour have been left blank for clarity.

gravitational mass, as a function of their true simulated values. These uncertainty estimates are obtained by marginalizing the joint distribution $p\left(z, M \mid M_{z}^{\prime},\{P\}\right)$ over the total mass to obtain $p\left(z \mid M_{z},\{P\}\right)$ and over the redshift to get $p\left(M \mid M_{z},\{P\}\right)$. From these marginalized distributions, we compute our representative uncertainties as half of the minimum interval to contain $68 \%$ of the total probability (analogous to the $1 \sigma$ uncertainty for a Gaussian distribution).

We observe in Table IV that the general trend in the accuracy of redshift estimation is relatively insensitive to total system mass, but as we would expect, we see a deterioration in accuracy for more distant sources. The same can be seen in Table $\mathrm{V}$ for the estimation of the total gravitational mass. A notable feature of our results is that the redshift and mass accuracies are clearly dominated by the measurement of the second feature at low masses and by the first feature at higher masses. One might expect the more localized first feature to always dominate. However, the ability to infer a mass from a redshifted frequency measurement using the relationship between the gravitational mass and the spectral feature frequency [Eq. (6)] is sensitive to the gradient of that relation in addition to the SNR of each feature. For lower masses, it is clear from Fig. 3 that mass measurements inferred from uncertain frequency measurements will have correspondingly larger uncertainties when the gradient of $f_{j}(M)$ is lower. This is the case for the first spectral feature at lower masses and the reason why this analysis requires numerical waveform simulations with such closely spaced mass values. 
TABLE IV. The percentage measurement uncertainties on the redshift of a BNS source. We show results for each of our five different mass systems and for each of four different redshifts. We give three fractional redshift uncertainties for each combination. The first and second entries correspond to results using the inspiral measurement plus the first and second spectral features, respectively. The third result (in bold face) is from a combination of the inspiral measurement and both spectral features. We have performed analyses of 100 different noise realizations, source, and sky orientations for each redshift and mass combination. For each realization, we compute a quantity equal to half of the span of the $68 \%$ confidence region on the redshift measurement. The quoted value is the median of this quantity over the 100 realizations.

\begin{tabular}{|c|c|c|c|c|c|c|c|c|c|c|c|c|c|c|c|}
\hline \multirow{3}{*}{$\frac{z}{0.01}$} & \multicolumn{15}{|c|}{ Total gravitational mass $M\left(M_{\odot}\right)$} \\
\hline & \multicolumn{3}{|c|}{2.6827} & \multicolumn{3}{|c|}{2.7567} & \multicolumn{3}{|c|}{2.8325} & \multicolumn{3}{|c|}{2.9101} & \multicolumn{3}{|c|}{2.9781} \\
\hline & 39.1 & 7.8 & 7.5 & 24.6 & 10.6 & 9.7 & 13.5 & 11.2 & 8.5 & 9.6 & 13.8 & 7.9 & 9.4 & 20.7 & 8.7 \\
\hline 0.02 & 40.4 & 10.0 & 9.2 & 25.7 & 13.9 & 11.6 & 17.5 & 16.5 & 11.9 & 11.6 & 20.6 & 10.1 & 13.4 & 32.8 & 12.0 \\
\hline 0.03 & 37.5 & 13.6 & 11.6 & 28.1 & 17.1 & 14.2 & 18.8 & 21.3 & 14.7 & 15.0 & 25.0 & 13.0 & 29.1 & 36.4 & 19.6 \\
\hline 0.04 & 31.8 & 15.3 & 14.9 & 26.8 & 19.1 & 15.8 & 21.2 & 23.5 & 15.7 & 18.6 & 26.4 & 15.1 & 23.7 & 30.5 & 19.4 \\
\hline
\end{tabular}

TABLE V. The percentage measurement uncertainties on the total gravitational mass of a BNS source. We show results for each of our five different mass systems and for each of four different redshifts. The details are identical to those given in Table IV.

\begin{tabular}{|c|c|c|c|c|c|c|c|c|c|c|c|c|c|c|c|}
\hline \multirow{3}{*}{$\frac{z}{0.01}$} & \multicolumn{15}{|c|}{ Total gravitational mass $M\left(M_{\odot}\right)$} \\
\hline & \multicolumn{3}{|c|}{2.6827} & \multicolumn{3}{|c|}{2.7567} & \multicolumn{3}{|c|}{2.8325} & \multicolumn{3}{|c|}{2.9101} & \multicolumn{3}{|c|}{2.9781} \\
\hline & 0.38 & 0.07 & 0.06 & 0.24 & 0.10 & 0.09 & 0.13 & 0.10 & 0.07 & 0.09 & 0.13 & 0.07 & 0.08 & 0.20 & 0.07 \\
\hline 0.02 & 0.78 & 0.18 & 0.17 & 0.50 & 0.26 & 0.22 & 0.33 & 0.32 & 0.22 & 0.21 & 0.39 & 0.18 & 0.24 & 0.63 & 0.22 \\
\hline 0.03 & 1.06 & 0.38 & 0.33 & 0.80 & 0.48 & 0.40 & 0.53 & 0.62 & 0.41 & 0.42 & 0.73 & 0.36 & 0.79 & 1.04 & 0.55 \\
\hline 0.04 & 1.24 & 0.61 & 0.58 & 1.03 & 0.72 & 0.60 & 0.80 & 0.93 & 0.60 & 0.68 & 0.98 & 0.55 & 0.87 & 1.11 & 0.71 \\
\hline
\end{tabular}

\section{CONCLUSIONS}

A well-known problem of the detection of $\mathrm{GW}$ from compact-object binaries at cosmological distances is the so-called mass-redshift degeneracy, namely, that GW measurements allow the determination of the redshifted mass $M_{z}=M(1+z)$, but not the gravitational mass $M$ or the redshift $z$ separately. GW observations allow the measurement of the luminosity distance, but this degeneracy restricts the cosmological application of GW observations, since it is the relation between the source's luminosity distance and its redshift that allows us to probe cosmological models. Until recently, it was thought that coincident EM and GW observations would be required to break the mass-redshift degeneracy, as EM observations would allow the host galaxy to be identified and the source's redshift measured. In this paper, we describe a novel approach to this problem that does not require an EM counterpart but does anticipate knowledge of the NS EOS and exploits instead the information encoded in the HMNS stage of a BNS waveform to break the mass-redshift degeneracy.

We describe how, with the use of five numerically generated BNS waveforms of very slightly differing mass, we are able to construct frequency-domain power-spectrum reference templates. The templates are designed to capture the evolution of two primary spectral features in the HMNS stage of the waveforms, as a function of the total gravitational mass. The characteristic frequencies of these spectral features are then fitted to polynomial functions of mass providing us with an ad hoc approximation to the characteristic frequencies for any mass. A Bayesian inference method is used to test the ability of the ET to measure the characteristic frequencies in the HMNS stage of the signal. These frequency measurements are coupled with our precomputed, empirical, frequency-mass relation and a measurement of the redshifted mass from the inspiral phase of the signal, allowing us to determine both the redshift and gravitational mass separately.

We have shown that in an analysis based on the signal's power spectrum, and ignoring all phase information within the HMNS stage, the measurement uncertainties in the redshift of sources at $z=0.01-0.04$ are $\sim 10-20 \%$, over the full range of simulated system masses. In addition, we find that the gravitational mass can be measured with fractional accuracies of $<1 \%$ in all cases. The SNR of the signals used in our simulation (after high-pass filtering and windowing) have mean (maximum) values ranging from 5.5 (12) for $z=0.04$ to 23 (50) for $z=0.01$ [48].

We stated earlier that the use of GW observations for astrophysical measurements such as the masses and radii of NSs requires precision measurements of both the luminosity distance and the intrinsic mass of the source. For the former, it has been correctly approximated that for BNS sources seen as coincident with gamma-ray bursts, the fractional uncertainty in luminosity distance is approximately equal to the inverse of the SNR [10,12]. However, in general, as shown in Ref. [11], strong correlations with the inclination angle of the binary can 
lead to luminosity distance uncertainties of $\sim 10 \%-60 \%$. Concerning the measurement of the intrinsic mass of the binary, the breaking of the redshift-mass degeneracy in our results highlights the problem of accurate gravitational mass measurements for the advanced detectors. Without the potentially rare EM identification of a host galaxy, advanced detectors have access to only the redshifted mass, which if used uncorrected will result in a systematic bias in the measurement of total mass of up to 5\% for advanced and $>100 \%$ for proposed third-generation detectors.

We have specifically ignored the tidal effects in the late inspiral phase that have been previously shown to be useful in redshift measurements. This choice was made to simplify our analysis and clearly identify the potential of this new technique. It is encouraging to find that both approaches have comparable redshift sensitivities of $\sim \mathcal{O}(10 \%)$, implying that a combination of their results will improve the overall redshift estimate.

Under the expectation that there is a single universal NS EOS, it is highly likely that by the time of the ET the NS EOS will be tightly constrained via various observations, including direct $\mathrm{GW}$ detections from the advanced GW detectors. We limit our study to a single EOS, but based on previous studies $[32,49]$, we expect that the general result holds well for all realistic possibilities. Of primary interest here is the general concept that there exists an additional "matter effect" found in the HMNS stage of the waveform that can provide frequency markers from which redshift information can be obtained.

We stress that this analysis is one of the first attempts to perform parameter estimation on the HMNS stage of BNS signals. While the numerically generated waveforms and Bayesian parameter estimation techniques used here represent current levels of sophistication in both fields, our analytic signal model approximation and mass-frequency fitting are necessarily simplistic. We expect that prior to the era of third-generation GW detectors, the understanding of BNS mergers through numerical relativity and direct GW detections will enable us to significantly enhance our $a d$ hoc models. In the future, a significant improvement in the accuracies of redshift measurements using the HMNS stage could become possible if a realistic model of the phase evolution were constructed. In such a scenario, an analysis of the type presented here may be applicable to signals found in the advanced GW detectors.

\section{ACKNOWLEDGMENTS}

We acknowledge useful discussions with our colleagues from the LSC-Virgo Collaboration, and C.M., S. G., and B. S. S. especially thank C. Ott, J. Read, and J. Veitch. C. M. and B.S.S. were funded by the Science and Technology Facilities Council (STFC) Grant No. ST/J000345/1, and S. G. is partially supported by NSF Grants No. PHY-1151197 and No. 1068881. Partial support comes from the DFG Grant SFB/Transregio 7 and by "NewCompStar," COST Action
MP1304. K. T. is supported by the LOEWE-Program in HIC for FAIR. The simulations were performed on SuperMUC at LRZ-Munich, on Datura at AEI-Potsdam, and on LOEWE at CSC-Frankfurt.

[1] G. M. Harry and LIGO Scientific Collaboration, Advanced LIGO: The Next Generation of Gravitational Wave Detectors, Classical Quantum Gravity 27, 084006 (2010).

[2] F. Acernese et al. (Virgo Collaboration), Advanced Virgo Baseline Design, Technical Report No. VIR-0027A-09, 2009.

[3] Y. Aso, Y. Michimura, K. Somiya, M. Ando, O. Miyakawa, T. Sekiguchi, D. Tatsumi, and H. Yamamoto, Interferometer Design of the KAGRA Gravitational Wave Detector, Phys. Rev. D 88, 043007 (2013).

[4] J. Abadie et al. (LIGO Scientific Collaboration and Virgo Collaboration), Predictions for the Rates of Compact Binary Coalescences Observable by Ground-Based GravitationalWave Detectors, Classical Quantum Gravity 27, 173001 (2010).

[5] M. Punturo et al., The Third Generation of Gravitational Wave Observatories and Their Science Reach, Classical Quantum Gravity 27, 084007 (2010).

[6] B. Sathyaprakash, M. Abernathy, F. Acernese, P. Ajith, B. Allen, P. Amaro-Seoane, N. Andersson, S. Aoudia, K. Arun, P. Astone et al., Scientific Objectives of Einstein Telescope, Classical Quantum Gravity 29, 124013 (2012).

[7] B.F. Schutz, Determining the Hubble Constant from Gravitational Wave Observations, Nature (London) 323, 310 (1986).

[8] S. Nissanke, D. E. Holz, N. Dalal, S. A. Hughes, J. L. Sievers, and C. M. Hirata, Determining the Hubble Constant from Gravitational Wave Observations of Merging Compact Binaries, arXiv:1307.2638.

[9] D. E. Holz and S. A. Hughes, Using Gravitational-Wave Standard Sirens, Astrophys. J. 629, 15 (2005).

[10] B. S. Sathyaprakash, B. F. Schutz, and C. Van Den Broeck, Cosmography with the Einstein Telescope, Classical Quantum Gravity 27, 215006 (2010).

[11] S. Nissanke, D. E. Holz, S. A. Hughes, N. Dalal, and J.L. Sievers, Exploring Short Gamma-Ray Bursts as Gravitational-Wave Standard Sirens, Astrophys. J. 725, 496 (2010).

[12] W. Zhao, C. Van Den Broeck, D. Baskaran, and T. G. F. Li, Determination of Dark Energy by the Einstein Telescope: Comparing with CMB, BAO, and SNIa Observations, Phys. Rev. D 83, 023005 (2011).

[13] H.-Y. Chen and D. E. Holz, Gamma-Ray-Burst Beaming and Gravitational-Wave Observations, Phys. Rev. Lett. 111, 181101 (2013).

[14] B. D. Metzger and E. Berger, What is the Most Promising Electromagnetic Counterpart of a Neutron Star Binary Merger?, Astrophys. J. 746, 48 (2012).

[15] E. Nakar, Short-Hard Gamma-Ray Bursts, Phys. Rep. 442, 166 (2007).

[16] W. H. Lee and E. Ramirez-Ruiz, The Progenitors of Short Gamma-Ray Bursts, New J. Phys. 9, 17 (2007). 
[17] L. Rezzolla, B. Giacomazzo, L. Baiotti, J. Granot, C. Kouveliotou, and M. A. Aloy, The Missing Link: Merging Neutron Stars Naturally Produce Jet-like Structures and Can Power Short Gamma-Ray Bursts, Astrophys. J. 732, L6 (2011).

[18] T. Bode, R. Haas, T. Bogdanovic, P. Laguna, and D. Shoemaker, Relativistic Mergers of Supermassive Black Holes and their Electromagnetic Signatures, Astrophys. J. 715, 1117 (2010).

[19] B. D. Farris, Y. Tung Liu, and S. L. Shapiro, Binary Black Hole Mergers in Gaseous Environments: "Binary Bondi" and “Binary Bondi-Hoyle-Lyttleton'”Accretion, Phys. Rev. D 81, 084008 (2010).

[20] O. Zanotti, L. Rezzolla, L. Del Zanna, and C. Palenzuela, Electromagnetic Counterparts of Recoiling Black Holes: General Relativistic Simulations of Non-Keplerian Discs, Astron. Astrophys. 523, A8 (2010).

[21] C. Palenzuela, L. Lehner, and S. L. Liebling, Dual Jets from Binary Black Holes, Science 329, 927 (2010).

[22] P. Moesta, D. Alic, L. Rezzolla, O. Zanotti, and C. Palenzuela, On the Detectability of Dual Jets from Binary Black Holes, Astrophys. J. Lett. 749, L32 (2012).

[23] D. F. Chernoff and L. S. Finn, Gravitational Radiation, Inspiraling Binaries, and Cosmology, Astrophys. J. Lett. 411, L5 (1993).

[24] L. S. Finn, Binary Inspiral, Gravitational Radiation, and Cosmology, Phys. Rev. D 53, 2878 (1996).

[25] S. R. Taylor and J. R. Gair, Cosmology with the Lights Off: Standard Sirens in the Einstein Telescope Era, Phys. Rev. D 86, 023502 (2012).

[26] S. R. Taylor, J. R. Gair, and I. Mandel, Cosmology Using Advanced Gravitational-Wave Detectors Alone, Phys. Rev. D 85, 023535 (2012).

[27] W. Del Pozzo, Inference of Cosmological Parameters from Gravitational Waves: Applications to Second Generation Interferometers, Phys. Rev. D 86, 043011 (2012).

[28] É. É. Flanagan and T. Hinderer, Constraining Neutron-Star Tidal Love Numbers with Gravitational-Wave Detectors, Phys. Rev. D 77, 021502 (2008).

[29] C. Messenger and J. Read, Measuring a Cosmological Distance-Redshift Relationship Using Only Gravitational Wave Observations of Binary Neutron Star Coalescences, Phys. Rev. Lett. 108, 091101 (2012).

[30] L. Baiotti, B. Giacomazzo, and L. Rezzolla, Accurate Evolutions of Inspiralling Neutron-Star Binaries: Prompt and Delayed Collapse to a Black Hole, Phys. Rev. D 78, 084033 (2008).

[31] L. Rezzolla, L. Baiotti, B. Giacomazzo, D. Link, and J. A. Font, Accurate Evolutions of Unequal-Mass Neutron-Star Binaries: Properties of the Torus and Short GRB Engines, Classical Quantum Gravity 27, 114105 (2010).

[32] A. Bauswein and H.-T. Janka, Measuring Neutron-Star Properties via Gravitational Waves from Neutron-Star Mergers, Phys. Rev. Lett. 108, 011101 (2012).

[33] K. Takami, L. Rezzolla, and L. Baiotti, Spectral Properties of the Gravitational-Wave Emission from Hypermassive Neutron Stars (to be published), 2014; K. Takami, L. Rezzolla, and L. Baiotti, Constraining the Equation of State of Neutron Stars from Binary Mergers, Phys. Rev. Lett. 113, 091104, (2014).

[34] A. Bauswein, H.-T. Janka, K. Hebeler, and A. Schwenk, Equation-of-State Dependence of the Gravitational-Wave
Signal from the Ring-Down Phase of Neutron-Star Mergers, Phys. Rev. D 86, 063001 (2012).

[35] W. Del Pozzo, T. G. F. Li, M. Agathos, C. Van Den Broeck, and S. Vitale, Demonstrating the Feasibility of Probing the Neutron-Star Equation of State with Second-Generation Gravitational-Wave Detectors, Phys. Rev. Lett. 111, 071101 (2013).

[36] E. Bozzo, L. Stella, M. van der Klis, A. Watts, D. Barret, J. Wilms, P. Uttley, J. W. den Herder, and M. Feroci, The LOFT Mission: New Perspectives in the Research Field of (Accreting) Compact Objects, Eur. Phys. J. Web Conf. 64, 9002 (2014).

[37] D. Pollney, C. Reisswig, L. Rezzolla, B. Szilágyi, M. Ansorg, B. Deris, P. Diener, E. Nils Dorband, M. Koppitz, A. Nagar, and E. Schnetter, Recoil Velocities from EqualMass Binary Black-Hole Mergers: A Systematic Investigation of Spin-Orbit Aligned Configurations, Phys. Rev. D 76, 124002 (2007).

[38] L. Baiotti, I. Hawke, P. J. Montero, F. Löffler, L. Rezzolla, N. Stergioulas, J. A. Font, and E. Seidel, Three-Dimensional Relativistic Simulations of Rotating Neutron Star Collapse to a Kerr Black Hole, Phys. Rev. D 71, 024035 (2005).

[39] L. Rezzolla and O. Zanotti, Relativistic Hydrodynamics (Oxford University Press, Oxford, England, 2013).

[40] E. Schnetter, S. H. Hawley, and I. Hawke, Evolutions in 3D Numerical Relativity Using Fixed Mesh Refinement, Classical Quantum Gravity 21, 1465 (2004).

[41] E. Gourgoulhon, P. Grandclément, K. Taniguchi, J.-A. Marck, and S. Bonazzola, Quasiequilibrium Sequences of Synchronized and Irrotational Binary Neutron Stars in General Relativity: Method and Tests, Phys. Rev. D 63, 064029 (2001).

[42] S. Hild, S. Chelkowski, and A. Freise, Pushing towards the ET Sensitivity Using "Conventional" Technology, arXiv:0810.0604.

[43] Using the more recent ET-D design enhances the sensitivity in the region of interest $1-3 \mathrm{kHz}$ by $\approx 12 \%$.

[44] N. Stergioulas, A. Bauswein, K. Zagkouris, and H.-T. Janka, Gravitational Waves and Non-Axisymmetric Oscillation Modes in Mergers of Compact Object Binaries, Mon. Not. R. Astron. Soc. 418, 427 (2011).

[45] J. Skilling, Nested Sampling for General Bayesian Computation, Bayesian Anal. 1, 833 (2006).

[46] C. Cutler and É. E. Flanagan, Gravitational Waves from Merging Compact Binaries: How Accurately Can One Extract the Binary's Parameters from the Inspiral Waveform?, Phys. Rev. D 49, 2658 (1994).

[47] K. G. Arun, B. R. Iyer, B. S. Sathyaprakash, and P. A. Sundararajan, Parameter Estimation of Inspiralling Compact Binaries Using 3.5 Post-Newtonian Gravitational Wave Phasing: The Nonspinning Case, Phys. Rev. D 71, 084008 (2005).

[48] A calculation of the SNR for an optimally oriented system at $20 \mathrm{Mpc}$ for a single Advanced LIGO detector yields results consistent with those given in Table II of Ref. [32].

[49] A. Bauswein, H.-T. Janka, K. Hebeler, and A. Schwenk, Equation-of-State Dependence of the Gravitational-Wave Signal from the Ring-Down Phase of Neutron-Star Mergers, Phys. Rev. D 86, 063001 (2012). 